\title{
Spatiotemporal intermittency and chaotic saddles in the regularized long-wave equation
}

\author{
Erico L. Rempel, ${ }^{1,2, a)}$ Rodrigo A. Miranda, ${ }^{3}$ and Abraham C.-L. Chian ${ }^{2,3}$ \\ ${ }^{1}$ Department of Applied Mathematics and Theoretical Physics (DAMTP), University of Cambridge, \\ Cambridge CB3 OWA, United Kingdom \\ ${ }^{2}$ Institute of Aeronautical Technology (IEFM/ITA) and World Institute for Space Environment Research \\ (WISER), São José dos Campos, São Paulo 12228-900, Brazil \\ ${ }^{3}$ National Institute for Space Research (INPE) and World Institute for Space Environment Research \\ (WISER), P.O. Box 515, São José dos Campos, São Paulo 12227-010, Brazil
}

(Received 11 December 2008; accepted 22 June 2009; published online 17 July 2009)

\begin{abstract}
Transition to intermittent spatiotemporal chaos is studied in the regularized long-wave equation, a nonlinear model of shallow water waves. A mechanism for the onset of on-off spatiotemporal intermittency is explored. In this mechanism, the coupling of two chaotic saddles triggers random switching between phases of laminar and bursty behaviors. The average time between bursts as a function of the control parameter follows a power law typical of crisis transitions in chaotic systems. The degree of spatiotemporal disorder in the observed fluid patterns is quantified by means of the time-averaged spectral entropy for both chaotic attractors and chaotic saddles. The implications of these results to other fluid systems are discussed. (C) 2009 American Institute of Physics.
\end{abstract}

[DOI: 10.1063/1.3183590]

\section{INTRODUCTION}

Since the pioneering work of Lorenz, ${ }^{1}$ the understanding of turbulent motions has motivated the study of temporal chaos in nonlinear systems. Chaos theory can describe some phenomena related to turbulence, such as coexistence of regular and irregular motion, coexistence of coherence and incoherence, broadband power spectra, and intermittency. However, the lack of spatial information in systems described by a small number of coupled ordinary differential equations (ODEs) makes it hard to draw conclusions on their usefulness for the interpretation of the dynamics of real fluids.

The analysis of infinite-dimensional dynamical systems modeled by partial differential equations (PDEs) can provide a bridge between chaos theory and fluid dynamics. Such systems may exhibit a wealth of regimes, which include temporal chaos (TC) and spatiotemporal chaos (STC). In PDEs, we refer to TC whenever the patterns generated vary chaotically in time, but spatial coherence is preserved. In STC, the dynamics is chaotic in time and irregular in space. Sometimes, the TC and STC behaviors are referred to as STC and fully developed STC, respectively. ${ }^{2}$ In relation to turbulence, a comparatively small number of degrees of freedom are active in STC, so the system lacks a fully developed turbulent cascade. ${ }^{3}$ The present work focuses on a crisis transition from TC to STC in the driven-damped regularized long-wave equation (RLWE), which has been derived in the past as a model of shallow water waves, ${ }^{4-6}$ as well as drift waves in magnetized plasmas. ${ }^{7,8}$

One of the ubiquitous features of dissipative spatiotemporal systems is the presence of transient chaos. Spatiotemporally chaotic transients have received much attention re-

${ }^{\mathrm{a})}$ Electronic mail: rempel@ita.br. cently after numerical simulations, ${ }^{9,10}$ and laboratory experiments ${ }^{11-13}$ have shown that, at least for moderate Reynolds numbers, turbulence in pipe flows is transient and eventually the flow decays to the laminar state with the average duration of transients depending on the Reynolds number and amplitude of perturbations. Chaotic transients are due to chaotic saddles, which are nonattracting chaotic sets. ${ }^{14-18}$ The coupling of distinct chaotic saddles embedded in a chaotic attractor results in intermittent switching between transient states. ${ }^{16-22}$ Recently, the coupling of a temporally chaotic saddle (TCS) and a spatiotemporally chaotic saddle (STCS) was proposed as a mechanism for the onset of TC-STC intermittency right after a transition to STC. ${ }^{23,24}$ In the present paper, we use chaotic saddles to study the degree of spatiotemporal disorder in the fluid patterns in the RLWE. We will show that in the intermittent regime following the transition to STC, the average time between bursts as a function of the control parameter obeys a power law similar to crisis transitions in low-dimensional chaotic systems. $^{25,26}$

This paper is organized as follows. In Sec. II we present the model equation and its numerical solution. Next, in Sec. III the transition from TC to STC is discussed. The presence of chaotic saddles and their role in transient and intermittent spatiotemporal dynamics is analyzed. The conclusions are given in Sec. IV.

\section{THE RLWE}

The unidirectional propagation of long waves in fluids with small but finite amplitude in systems with nonlinearity and dispersion can be described by the Korteweg-de Vries $(\mathrm{KdV})$ equation, which in dimensionless form is given $\mathrm{by}^{5,6,27}$ 


$$
\partial_{t} u+\partial_{x} u+u \partial_{x} u+\partial_{x x x} u=0
$$

Equation (1) is often written without the second term after taking $x^{\prime}=x-t$ and $t$ as independent variables. The $\mathrm{KdV}$ equation was originally derived for water waves and has been used as a model for long waves in many other physical systems, ${ }^{6}$ e.g., nonlinear ion-acoustic waves in plasmas. ${ }^{27}$ However, some mathematical properties of the KdV equation, such as the existence and stability of solutions, are difficult to study. ${ }^{5}$ Furthermore, there are other problems mainly due to the dispersion term. For example, from the linear dispersion relation of Eq. (1),

$$
\omega=k-k^{3},
$$

one can observe that the phase velocity $V_{\mathrm{ph}}=\omega / k$ becomes negative for $k^{2}>1$, which is inconsistent with the assumption of forward-traveling waves used in the derivation of Eq. (1)..$^{5}$ Moreover, the group velocity

$$
\frac{d \omega}{d k}=1-3 k^{2},
$$

does not have any lower limit, thus there is no bound to the velocity with which short wavelength features propagate. These impediments have led to the pursuit of an alternative model. The RLWE was proposed by Peregrine ${ }^{4}$ and Benjamin et al. ${ }^{5}$ as an alternative to the $\mathrm{KdV}$ equation. It was later derived by $\mathrm{He}$ and $\mathrm{Salat}^{7}$ as a model for nonlinear drift waves in plasmas with a periodic driving term and a linear damping term introduced $a d$ hoc in order to study transition to chaos. The driven-damped RLWE is given by ${ }^{7,23,28-31}$

$$
\partial_{t} \phi+c \partial_{x} \phi+f \phi \partial_{x} \phi+a \partial_{t x x} \phi=-\nu \phi-\varepsilon \sin (x-\Omega t),
$$

where $a, c$, and $f$ are constants, $\nu$ is a damping parameter, $\varepsilon$ is the driver amplitude, and $\Omega$ is the driver frequency. Originally, Peregrine ${ }^{4}$ obtained Eq. (4) (without the two terms on the right-hand side) from the momentum equation for the mean horizontal velocity of water $\phi(x, t)$ of an irrotational flow by assuming that waves only travel in one direction and the ratios between wave amplitude and water depth and between water depth and wavelength are small, where wavelength means the distance in which significant changes in surface height occur. The third-order derivative term in Eq. (4) expresses the effect of the vertical acceleration of water on pressure. In the absence of forcing $(\varepsilon=0)$, the linear dispersion relation is

$$
\omega(k)=(i \nu-c k) /\left(a k^{2}-1\right),
$$

from which the phase velocity can be obtained

$$
V_{\mathrm{ph}}=-\frac{c}{a k^{2}-1}+\frac{i \nu}{k\left(a k^{2}-1\right)}
$$

Assuming that $a<0$ and $c>0, \operatorname{Re}\left[V_{\mathrm{ph}}\right]>0$ for all $k$. The group velocity

$$
V_{g}=-\frac{c}{a k^{2}-1}+2 a c k^{2}\left(\frac{1}{a k^{2}-1}\right)^{2}-2 i a \nu k\left(\frac{1}{a k^{2}-1}\right)^{2}
$$

approaches zero for large $k$, which means that high wavenumber features do not propagate. The existence, uniqueness, and stability of solutions of the RLWE have been formally demonstrated by Benjamin et al. ${ }^{5}$

We define periodic boundary conditions $\phi(x, t)$ $=\phi(x+2 \pi, t)$ and fix $a=-0.287, c=1, f=-6, \nu=0.1$, and $\Omega=0.65$. These parameter values can be chosen arbitrarily with the exception of $a$, which must be negative for physical reasons and to avoid numerical instability. ${ }^{7}$ Here, the values are chosen in order to study the transition to STC previously identified by $\mathrm{He}^{28}$ Thus, the driver amplitude $\varepsilon$ is the only control parameter. From Eq. (5), since $a<0$ and $\nu>0$, $\operatorname{Im}[\omega(k)]<0$ for all $k$. Thus, all modes are linearly damped. This is in contrast to the Kuramoto-Sivashinsky equation studied by Rempel et al., ${ }^{24}$ where there was a band of linearly unstable Fourier modes. In the case of the RLWE, the external driver is necessary to destabilize the mode with wave number $k=1$, as discussed below.

We solve Eq. (4) with the spectral (Galerkin) method by expanding $\phi(x, t)$ in a Fourier series ${ }^{23}$

$$
\phi(x, t)=\sum_{k=-N}^{N} b_{k}(t) e^{i k x},
$$

where $k=2 \pi n / L, n=-N, \ldots, N, L=2 \pi$ is the system length, and $i=\sqrt{-1}$. We set $N=32$. By introducing Eq. (8) into Eq. (4), one obtains a set of ODEs in terms of the complex Fourier coefficients $b_{k}(t)$,

$$
\begin{aligned}
\left(1-a k^{2}\right) \frac{d b_{k}}{d t}= & -i c k b_{k}-\nu b_{k}+\frac{\varepsilon}{2} \sin (\Omega t) \delta_{1, k} \\
& +\frac{i \varepsilon}{2} \cos (\Omega t) \delta_{1, k}-\left[i f k b_{k} b_{k}\right]_{k},
\end{aligned}
$$

where $\delta$ is the Kronecker delta and the last term on the right-hand side is the Fourier transform of $f \phi \partial_{x} \phi$, which is responsible for nonlinear wave coupling. In order to compute this term, the pseudospectral method is used, where the derivative is obtained in the Fourier space, $\partial_{x} \phi \rightarrow i k b_{k}$. Next, both $i k b_{k}$ and $b_{k}$ are inverse-Fourier transformed to real space, where the multiplication $f \phi \partial_{x} \phi$ is performed. Finally, the result is Fourier transformed again and is inserted into Eq. (9). Numerical integration is performed using the lsodar integrator, ${ }^{32}$ a variable-step integrator which is readily available. ${ }^{33}$ At each time step, 1/3 of the high $k$ modes are set to zero in order to avoid aliasing errors. ${ }^{34}$ Thus, the effective number of modes is $N=20$. From Eq. (9), it can be seen that in the presence of an external driver $(\varepsilon \neq 0)$, energy is injected into the mode $k=1$ and spreads toward other modes through the nonlinear term. Thus, the forcing and nonlinear terms are responsible for driving the system away from equilibrium and leading the system to chaos. 

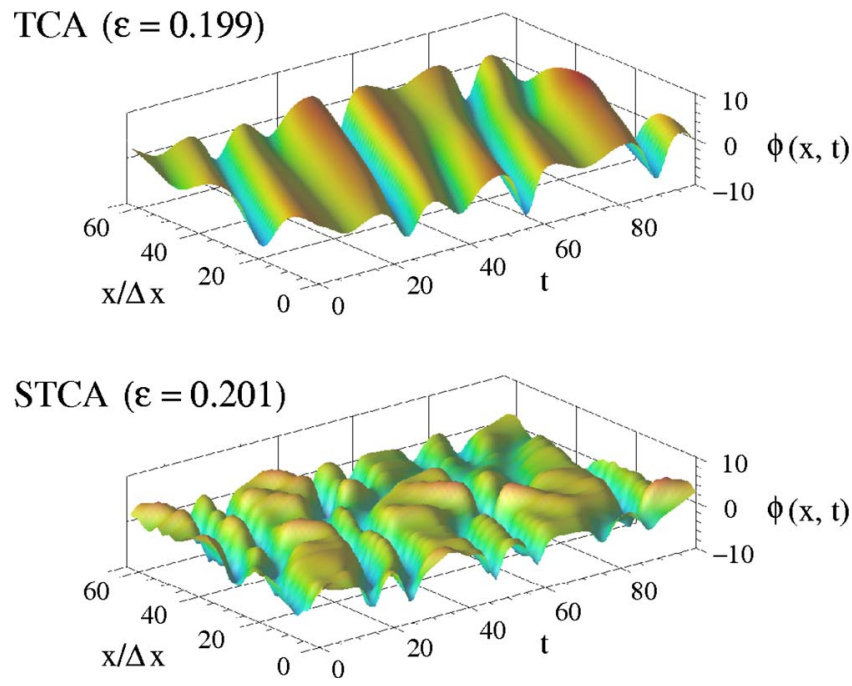

FIG. 1. (Color online) Spatiotemporal patterns $\phi(x, t)$ of the RLWE for $\varepsilon=0.199$ (TCA, upper panel) and $\varepsilon=0.201$ (STCA, lower panel).

\section{RESULTS}

\section{A. Transition to STC}

The transition to STC can be easily recognized as a sudden change in the spatiotemporal patterns of the numerical solutions of Eq. (4). Figure 1 shows the asymptotic solutions obtained for two values of the control parameter $\varepsilon$. The first regime (Fig. 1, upper panel) obtained for $\varepsilon=0.199$ is regular in space, but a positive value of the maximum Lyapunov exponent $\left(\lambda_{\max } \approx 0.05\right)$ for the attractor indicates that the dynamics is temporally chaotic (TC). The Lyapunov exponents are obtained from the eigenvalues of the linearized vector field along a trajectory, and are computed with the code given in Wolf et al., ${ }^{35}$ where a Gram-Schmidt orthonormalization process which employs the Euclidean norm is used and base 2 logarithm is adopted for the exponents. The lower panel of Fig. 1 shows that in the second regime $(\varepsilon=0.201)$ the spatiotemporal patterns are disordered in both space and time. The maximum Lyapunov exponent for the attractor STCA has jumped to $\lambda_{\max } \approx 0.12$. The transition from spatial regularity to spatial irregularity is due to an attractorwidening crisis that occurs after the collision of the spatially regular attractor with an unstable saddle orbit and has been described in a series of papers. ${ }^{28-30}$ The energy distribution among Fourier modes can be seen in the time-averaged power spectra $\left\langle\left|b_{k}\right|^{2}\right\rangle_{t}$ depicted in Fig. 2 for $N=32$ (left) and for $N=512$ (right). The spectrum in the temporally chaotic attractor (TCA) regime $(\varepsilon=0.199$, dashed line) is narrower than in the spatiotemporally chaotic attractor (STCA) regime $(\varepsilon=0.201$, solid line $)$. This indicates that at the onset of STC, when the spatial regularity is destroyed, spectral energy cascades to neighboring modes due to nonlinear wave-wave interactions, increasing the number of active modes. The energy spreading remains essentially the same for $N=32$ and $N=512$.

The amount of spatial disorder can be quantified by means of the spectral entropy ${ }^{24,36,37}$

$$
S(t)=-\sum_{k=1}^{N} p_{k}(t) \ln \left[p_{k}(t)\right]
$$

where $p_{k}(t)$ is the relative weight of a Fourier mode $k$ at an instant $t$

$$
p_{k}(t)=\frac{\left|b_{k}(t)\right|^{2}}{\sum_{k}\left|b_{k}(t)\right|^{2}}
$$

Since $\phi(x, t)$ in Eq. (4) is a real variable,

$$
\left|b_{-k}(t)\right|=\left|b_{k}(t)\right|,
$$

only Fourier modes with $k>0$ need to be considered. Note from Eq. (9) that the mode $b_{0}(t)$ is decoupled from the other modes and is null for all $t$ if $b_{0}(0)=0$. The spectral entropy is maximum for a random system with uniform distribution, i.e., for all $k, p_{k}(t)=1 / N$. In this case, $S(t)=\ln N .^{38}$ For $N=20$, the maximum entropy is $S_{\max } \approx 3$.

Figure 3 shows the variation in the time-averaged spectral entropy $\langle S\rangle_{t}$ for the attracting solutions (solid line) of Eq. (4) as a function of the control parameter $\varepsilon$. The onset of STC can be clearly seen as a sudden increase in the value of $\langle S\rangle_{t}$ at $\varepsilon \approx 0.2$. The other curves displayed in Fig. 3 are discussed in Sec. III B.
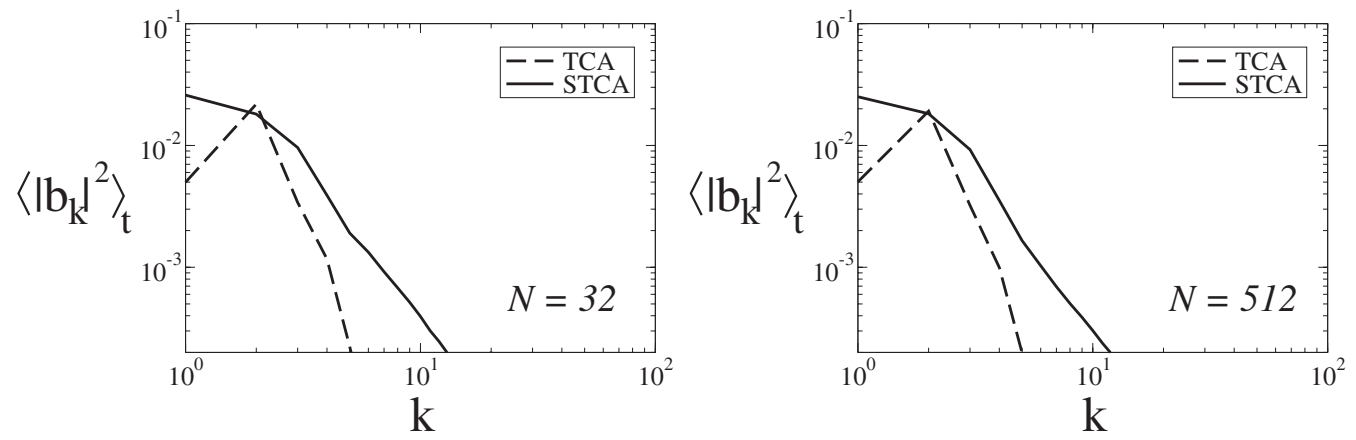

FIG. 2. Time-averaged power spectra in the $k$ wavenumber domain for $\varepsilon=0.199$ (TCA regime, dashed line) and $\varepsilon=0.201$ (STCA regime, solid line), for simulations with $N=32$ (left) and $N=512$ (right) Fourier modes. 


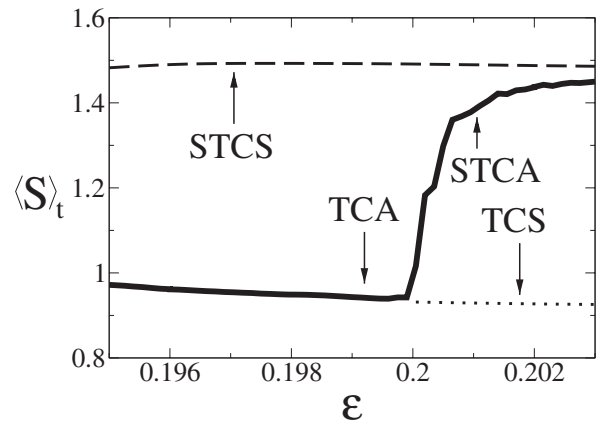

FIG. 3. Time-averaged spectral entropy as a function of the driver amplitude $\varepsilon$. The solid line represents the attractors (TCA and STCA), the dashed line denotes the STCS, and the dotted line the TCS.

\section{B. Transient and intermittent spatiotemporal dynamics}

By neglecting the dissipation and forcing terms on the right-hand side of Eq. (4), after multiplication by $\phi$ and integration in the spatial domain, one can obtain an equation to describe the temporal evolution of the "wave energy," which is a constant of motion for $\nu=\varepsilon=0$, , 31,39

$$
E(t)=\frac{1}{4 \pi} \int_{0}^{2 \pi}\left[\phi^{2}-a \phi_{x}^{2}\right] d x .
$$

We use the time series of the wave energy to identify transient and intermittent behaviors in the RLWE. Another quantity that has proven useful in this task is the time series for the height of the main peak of the power spectrum $h .^{24}$ Figure 4 shows the time series for the wave energy $E$ and the main peak height $h$. In the TC regime (Fig. 4, left panels) the time series of $E$ and $h$ display an initial behavior of highlevel fluctuations before converging asymptotically to a "laminar" state with lower variability, corresponding to the TCA of Fig. 1 (upper panel). In the STC regime (Fig. 4, right panels) the time series of $E$ and $h$ display intermittent switchings between "bursty" (high variability) and laminar (low
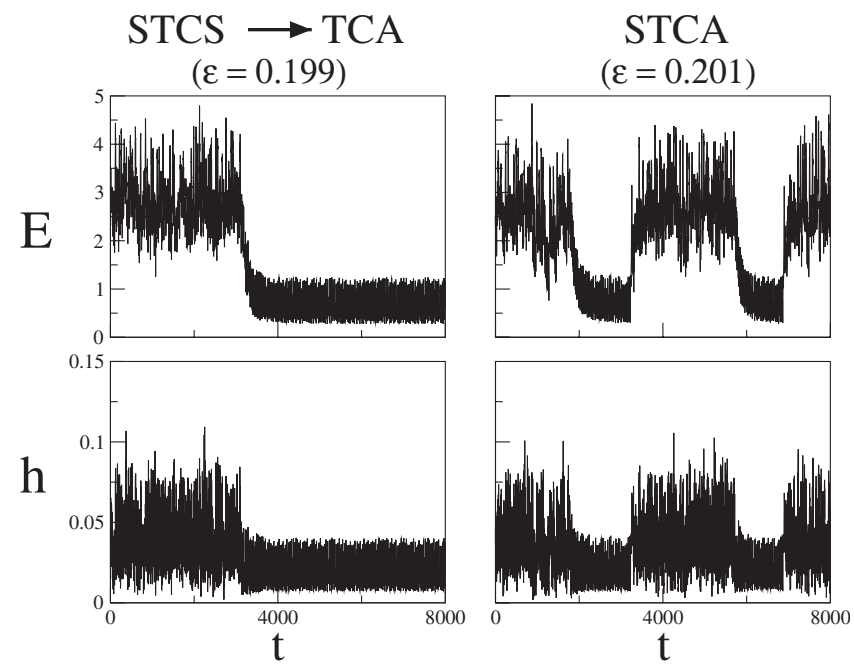

FIG. 4. Time series of wave energy $E$ (upper panels) and the maximum peak value of power spectrum $h$ (lower panels) for the RLWE in the TC ( $\varepsilon=0.199$, left panels $)$ and STC $(\varepsilon=0.201$, right panels $)$ regimes. In the TC regime, there is transient STC due to a STCS.
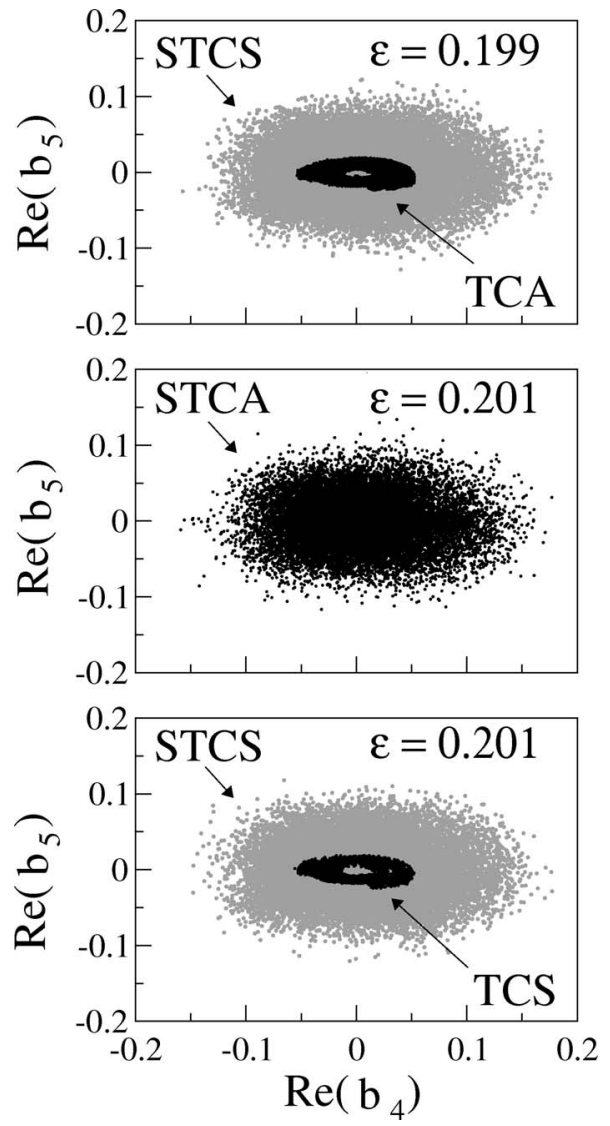

FIG. 5. Projections of attracting and nonattracting chaotic sets for the RLWE for $\varepsilon=0.199$ (upper panel), showing the TCA (black) and the STCS (gray), and for $\varepsilon=0.201$ (middle panel), showing the STCA, which is decomposed in the lower panel into a TCS (black) and a STCS (gray).

variability) behaviors. It is important to distinguish the nature of this TC-STC intermittency found in the RLWE from the spatiotemporal intermittency reported by Chaté and Manneville, ${ }^{40}$ where patches of laminar and bursty behaviors coexist in space. Here the bursty phases are clearly localized in time but extend over the whole space, similar to the spatiotemporal intermittency found in the damped KuramotoSivashinsky equation with periodic boundary conditions ${ }^{24}$ and experiments with liquid columns hanging below an overflowing circular dish. ${ }^{41}$

As mentioned before, chaotic transients are due to the presence of chaotic saddles in the phase space. We adopt a Poincaré map in which a point is plotted every time the flow of Eq. (9) crosses the plane $\operatorname{Re}\left[b_{1}(t)\right]=0$ with $\mathrm{d}\left\{\operatorname{Re}\left[b_{1}(t)\right]\right\} / d t>0$. Then, we employ the sprinkler method $^{14,15}$ to find chaotic saddles. In Fig. 5 the attracting and nonattracting chaotic sets are represented as projections of the Poincaré points on the $\left[\operatorname{Re}\left(b_{4}\right), \operatorname{Re}\left(b_{5}\right)\right]$ plane. At $\varepsilon=0.199$, prior to the onset of STC, it is possible to find a chaotic saddle coexisting with the TCA. This chaotic saddle is responsible for transient STC and is duly named STCS. ${ }^{23,24}$ Figure 5 (upper panel) depicts the TCA (black) and the STCS (gray) for $\varepsilon=0.199$. The latter surrounds the region occupied by the TCA. After the transition to STC $[\varepsilon=0.201$, Fig. 5 (middle panel)], the attractor expands abruptly to include the region previously occupied by the 


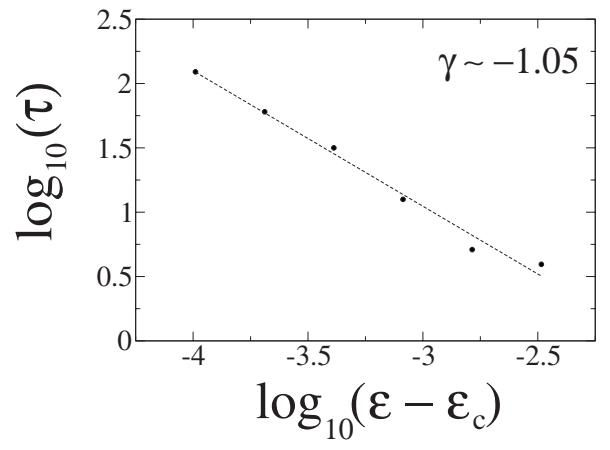

FIG. 6. Average duration of laminar intervals $\tau$ as a function of the departure from the critical value of the control parameter $\left(\varepsilon_{c}=0.2\right)$ in the $\log -\log$ scale for the RLWE. The straight line shows a least-squares fit with slope $\gamma \sim-1.05$.

STCS. If one applies the sprinkler method in this regime, two chaotic saddles embedded in the STCA can be found. They are shown in Fig. 5, where the STCA (middle panel) is decomposed into a STCS (gray) and a TCS (black) (lower panel) which evolves from the destabilized TCA. In the sprinkler method, the chaotic saddle is approximated by points from trajectories that follow long transients before escaping from a predefined restraining region of the phase space. Given the difficulty in defining a restraining region in a high-dimensional phase space, we use the different levels of wave energy displayed by the TC and STC regimes to identify the "restraining regions." To find the STC saddle, a large set of initial conditions is iterated and those trajectories for which $E(t)>1.2$ for 100 consecutive iterations of the Poincaré map $(t \approx 1000)$ are considered to be in the vicinity of the STC saddle. For each of those trajectories, the first 40 and last 40 iterations are discarded and only 20 points are plotted. For the TC saddle, the restraining region is defined as above, but with $E(t)<1.2$ instead. This threshold of $E(t)$ is found after an inspection of the variability of $E(t)$ in Fig. 4. The number of iterations discarded is chosen after some trial and error (see Ref. 15).

The attractor decomposition mentioned in the previous paragraph suggests that chaotic saddles dominate the spatiotemporal intermittent dynamics found after the onset of STC. Their signatures can be seen in the time series shown in Fig. 4. The STCS governs the dynamics of the initial transient in the left panels (TC regime) and the bursty periods in the right panels (STC regime). Moreover, the similarity between the TCA regime before the STC transition and the laminar phases after the STC transition is due to the fact that, after the onset of STC, the TCA loses its stability, becoming a TCS, which governs the laminar periods in the STCA regime. The TC-STC intermittency consists of random switchings between phases where the dynamics is basically governed by TCS and STCS, respectively.

As the control parameter $\varepsilon$ is increased beyond the transition point, the average duration of the laminar periods $\tau$ decreases as a power law. In Fig. 6 we plot $\tau$ as a function of the departure from the critical value of the control parameter (here taken as $\left.\varepsilon_{c}=0.2\right)$ in the $\log$-log scale. This result indicates that the STCS dominates the spatiotemporal dynamics of the STCA when the value of the control parameter $\varepsilon$ is increased. This can also be seen in Fig. 3, where the values of the time-averaged spectral entropy for STCS and TCS are represented by the dashed and dotted lines, respectively. The spectral entropy of STCA rapidly approaches the entropy of STCS after the transition at $\varepsilon_{c} \approx 0.2$. It is clear that the spectral entropy of STCS for $\varepsilon<\varepsilon_{c}$ can be used to predict the dynamics in STCA for $\varepsilon>\varepsilon_{c}$, as pointed out in Ref. 23.

\section{CONCLUSIONS}

In this paper, we have demonstrated that chaotic saddles are responsible for chaotic transients and intermittency in a nonlinear model of long waves in shallow waters. When the dynamics is regular in space and chaotic in time, a STCS dominates the transient dynamics. After the transition from temporal chaos to STC due to a crisis, the attractor undergoes a sudden increase in size and complexity in both space and time, reflected by a broader power spectrum and an increase in spectral entropy and the maximum Lyapunov exponent. The TC-STC intermittency in this regime is caused by the coupling of two chaotic saddles embedded in the enlarged attractor. One is a continuation of the STCS responsible for transient chaos prior to the crisis and the other is a continuation of the spatially regular TCA (now turned into TCS). We believe that this coupling is due to the sudden creation of a set of coupling unstable periodic orbits (UPOs) at the crisis (related to "explosion"16-18,42). It has been shown for low-dimensional dynamical systems ${ }^{16-19,43}$ that in a Poincaré map these coupling unstable orbits possess points in the regions of the phase space occupied by both chaotic saddles, and the coupling is done through a series of heteroclinic connections between the manifolds of the coupling UPOs and the manifolds of the UPOs that belong to the chaotic saddles.

Although the spatial resolution of the numerical solution of the RLWE used in this work is small, the dynamics is essentially the same for higher resolutions. This is a consequence of the fact that for the selected parameters only a small number of modes are active in the pre- and posttransition dynamics, so the system corresponds to weak wave turbulence. ${ }^{44,45}$

The results presented here are consistent with those reported by Rempel et al. ${ }^{24}$ where the damped KuramotoSivashinsky equation with periodic boundary conditions was studied. Hence, we suggest that the mechanism for the onset of TC-STC intermittency via the coupling of chaotic saddles can be readily found in other fluid systems in transition from laminar to weakly turbulent flows provided the following conditions are met: (1) there is a discontinuous transition from temporal chaos to STC due to a crisislike phenomenon and (2) before the transition, the system displays transient STC. Examples of fluids with chaotic transients and a crisis transition to STC or turbulence may include the onset of bursting behavior in a driven, two-dimensional viscous flow subject to no-slip boundaries ${ }^{46}$ and the onset of turbulence in pipe flows. In the latter case, a boundary crisis seems to be responsible for converting a transient turbulent state into a 
turbulent attractor at Reynolds number of $\sim 1800 .{ }^{10,11} \mathrm{Al}-$ though recent experiments seem to indicate that turbulence in pipe flows is indeed a transient phenomenon, ${ }^{12,13}$ thus contradicting the previous results. Whether it is transient for all Reynolds numbers or there is a crisis bifurcation to an attractor remains an open question. ${ }^{47}$

\section{ACKNOWLEDGMENTS}

This work was supported by CNPq (Brazil) and FAPESP (Brazil). E.L.R. thanks Professor M. R. E. Proctor and DAMTP for their kind hospitality.

${ }^{1}$ E. Lorenz, "Deterministic nonperiodic flow," J. Atmos. Sci. 20, 130 (1963).

${ }^{2}$ T. Tél and Y.-C. Lai, "Chaotic transients in spatially extended systems," Phys. Rep. 460, 245 (2008).

${ }^{3}$ N. T. Ouellette and J. P. Gollub, "Dynamic topology in spatiotemporal chaos," Phys. Fluids 20, 064104 (2008).

${ }^{4}$ D. H. Peregrine, "Calculations of the development of an undular bore," J. Fluid Mech. 25, 321 (1966).

${ }^{5}$ T. B. Benjamin, J. L. Bona, and J. J. Mahony, "Model equations for long waves in nonlinear dispersive systems," Philos. Trans. R. Soc. London, Ser. A 272, 47 (1972).

${ }^{6}$ R. K. Dodd, J. C. Eilbeck, J. D. Gobbon, and H. C. Morris, Solitons and Nonlinear Wave Equations (Academic, London, 1982).

${ }^{7} \mathrm{~K}$. He and A. Salat, "Hysteresis and onset of chaos in periodically driven nonlinear drift waves," Plasma Phys. Controlled Fusion 31, 123 (1989).

${ }^{8}$ W. Horton, "Nonlinear drift waves and transport in magnetized plasma," Phys. Rep. 192, 1 (1990).

${ }^{9}$ H. Faisst and B. Eckhardt, "Sensitive dependence on initial conditions in transition to turbulence in pipe flow," J. Fluid Mech. 504, 343 (2004).

${ }^{10} \mathrm{~A}$. P. Willis and R. R. Kerswell, "Critical behavior in the relaminarization of localized turbulence in pipe flow," Phys. Rev. Lett. 98, 014501 (2007).

${ }^{11}$ J. Peixinho and T. Mullin, "Decay of turbulence in pipe flow," Phys. Rev. Lett. 96, 094501 (2006).

${ }^{12}$ B. Hof, J. Westerweel, T. M. Schneider, and B. Eckhardt, "Finite lifetime of turbulence in shear flows," Nature (London) 443, 59 (2006).

${ }^{13}$ B. Hof, A. de Lozar, D. J. Kuik, and J. Westerweel, "Repeller or attractor? Selecting the dynamical model for the onset of turbulence in pipe flow," Phys. Rev. Lett. 101, 214501 (2008).

${ }^{14} \mathrm{H}$. Kantz and P. Grassberger, "Repellers, semi-attractors, and long-lived chaotic transients," Physica D 17, 75 (1985).

${ }^{15}$ G.-H. Hsu, E. Ott, and C. Grebogi, "Strange saddles and the dimensions of their invariant manifolds," Phys. Lett. A 127, 199 (1988).

${ }^{16}$ E. L. Rempel, A. C.-L. Chian, E. E. N. Macau, and R. R. Rosa, "Analysis of chaotic saddles in low-dimensional dynamical systems: The derivative nonlinear Schrödinger equation," Physica D 199, 407 (2004).

${ }^{17}$ E. L. Rempel and A. C.-L. Chian, "Alfvén chaotic saddles," Int. J. Bifurcation Chaos Appl. Sci. Eng. 14, 4009 (2004).

${ }^{18}$ A. C.-L. Chian, W. M. Santana, E. L. Rempel, F. A. Borotto, T. Hada, and Y. Kamide, "Chaos in driven Alfvén systems: Unstable periodic orbits and chaotic saddles," Nonlinear Processes Geophys. 14, 17 (2007).

${ }^{19}$ K. G. Szabó, Y.-C. Lai, T. Tél, and C. Grebogi, "Topological scaling and gap filling at crisis," Phys. Rev. E 61, 5019 (2000).

${ }^{20}$ E. L. Rempel and A. C.-L. Chian, "High-dimensional chaotic saddles in the Kuramoto-Sivashinsky equation," Phys. Lett. A 319, 104 (2003).

${ }^{21}$ E. L. Rempel, A. C.-L. Chian, E. E. N. Macau, and R. R. Rosa, "Analysis of chaotic saddles in high-dimensional dynamical systems: The Kuramoto-Sivashinsky equation," Chaos 14, 545 (2004).

${ }^{22}$ E. L. Rempel and A. C.-L. Chian, "Intermittency induced by attractormerging crisis in the Kuramoto-Sivashinsky equation," Phys. Rev. E 71, 016203 (2005).

${ }^{23}$ E. L. Rempel and A. C.-L. Chian, "Origin of transient and intermittent dynamics in spatiotemporal chaotic systems," Phys. Rev. Lett. 98, 014101 (2007).

${ }^{24}$ E. L. Rempel, A. C.-L. Chian, and R. A. Miranda, "Chaotic saddles at the onset of intermittent spatiotemporal chaos," Phys. Rev. E 76, 056217 (2007).

${ }^{25}$ C. Grebogi, E. Ott, F. Romeiras, and J. A. Yorke, "Critical exponents for crisis-induced intermittency," Phys. Rev. A 36, 5365 (1987).

${ }^{26}$ A. C.-L. Chian, Complex Systems Approach to Economic Dynamics (Springer, Heidelberg, 2007).

${ }^{27}$ D. R. Nicholson, Introduction to Plasma Theory (Wiley, New York, 1983).

${ }^{28} \mathrm{~K}$. He, "Crisis-induced transition to spatiotemporally chaotic motions," Phys. Rev. Lett. 80, 696 (1998).

${ }^{29} \mathrm{~K}$. He and A. C.-L. Chian, "Critical dynamic events at the crisis of transition to spatiotemporal chaos," Phys. Rev. E 69, 026207 (2004).

${ }^{30} \mathrm{~K}$. He and A. C.-L. Chian, "On-off collective imperfect phase synchronization and bursts in wave energy in a turbulent state," Phys. Rev. Lett. 91, 034102 (2003).

${ }^{31} \mathrm{~K}$. He and A. C.-L. Chian, "Nonlinear dynamics of turbulent waves in fluids and plasmas," Nonlinear Processes Geophys. 12, 13 (2005).

${ }^{32}$ L. R. Petzold, "Automatic selection of methods for solving stiff and nonstiff systems of ordinary differential equations," SIAM (Soc. Ind. Appl. Math.) J. Sci. Stat. Comput. 4, 136 (1983).

${ }^{33}$ See the Netlib repository of the Oak Ridge National Laboratory (www.netlib.org) for information on the lsodar integrator.

${ }^{34}$ T. Tajima, Computational Plasma Physics: With Applications to Fusion and Astrophysics (Addison-Wesley, Redwood City, CA, 1989).

${ }^{35}$ A. Wolf, J. B. Swift, H. L. Swinney, and J. A. Vastano, "Determining Lyapunov exponents from a time series," Physica D 16, 285 (1985).

${ }^{36}$ G. E. Powell and I. C. Percival, "A spectral entropy method for distinguishing regular and irregular motion of Hamiltonian systems," J. Phys. A 12, 2053 (1979).

${ }^{37} \mathrm{H}$. Xi and J. D. Gunton, "Spatiotemporal chaos in a model of RayleighBénard convection,” Phys. Rev. E 52, 4963 (1995).

${ }^{38}$ R. Badii and A. Politi, Complexity: Hierarchical Structures and Scaling in Physics (Cambridge University Press, Cambridge, 1997).

${ }^{39}$ T. B. Benjamin, "The stability of solitary waves," Proc. R. Soc. London, Ser. A 328, 153 (1972).

${ }^{40} \mathrm{H}$. Chaté and P. Manneville, "Transition to turbulence via spatiotemporal intermittency,” Phys. Rev. Lett. 58, 112 (1987).

${ }^{41}$ P. Brunet and L. Limat, "Defects and spatiotemporal disorder in a pattern of falling liquid columns," Phys. Rev. E 70, 046207 (2004).

${ }^{42}$ C. Robert, K. T. Alligood, E. Ott, and J. A. Yorke, "Explosions of chaotic sets," Physica D 144, 44 (2000).

${ }^{43}$ K. G. Szabó, Y.-C. Lai, T. Tél, and C. Grebogi, "Critical exponent for gap filling at crisis," Phys. Rev. Lett. 77, 3102 (1996).

${ }^{44} \mathrm{P}$. Manneville, Dissipative Structures and Weak Turbulence (Academic, New York, 1990).

${ }^{45}$ M. M. Degen, I. Mutabazi, and C. D. Andereck, "Transition to weak turbulence via spatiotemporal intermittency in the Taylor-Dean system," Phys. Rev. E 53, 3495 (1996).

${ }^{46}$ D. Molenaar, H. J. H. Clercx, and G. J. F. van Heijst, "Attractor crisis and bursting in a fluid flow with two no-slip directions," Phys. Rev. E 75, 036309 (2007)

${ }^{47}$ B. Eckhardt, "Turbulence transition in pipe flow: Some open questions," Nonlinearity 21, T1 (2008). 